\title{
GEOPOLITICA CONTEMPORÁNEA
}

\author{
Y ANÁLISIS DE FACTORES \\ RELEVANTESA ESCALA GLOBAL*
}

Gustavo Ferro Núñez ${ }^{(a)}$, Óscar Andrés Castaño Ferro ${ }^{(b)}$

CONTEMPORARY GEOPOLITICS AND ANALYSIS OF

RELEVANT FACTORS ON A GLOBAL SCALE

GEOPOLÍTICA CONTEMPORÂNEA E ANÁLISE DE

FATORES RELEVANTES A ESCALA GLOBAL

Fecha de recepción: 10 de agosto del 2016

Fecha de aprobación: 24 de agosto del 2017

Sugerencia de citación:

Ferro Nuñez, G. y Castaño Ferro, Ó. A. (2017). Geopolítica contemporánea y análisis de factores relevantes a escala global. Razón Crítica, 3, 111-144, doi: http://dx.doi.org/10.21789/25007807.1235

* El presente artículo es un ejercicio realizado siguiendo la metodología de revisión bibliográfica y análisis temático especializado. Se elaboró en el 2016 como parte de una labor investigativa más amplia.

(a) Graduado en Mercadeo de la Universidad de Ibagué, Especialista en Docencia universitaria del convenio Universidad de La Habana- Universidad de Ibagué, Magíster en Administración de Negocios internacionales del Convenio Instituto tecnológico de estudios superiores de MonterreyUniversidad Autónoma de Bucaramanga y Doctorado en Planeación estratégica de la Universidad del Atlántico de EE. UU. Miembro del grupo de investigación GIPE de la Universidad del Tolima. Profesor de planta de la Universidad del Tolima, Ibagué, Colombia http:/ / orcid.org/0000-0002-7354-9382. gferron@ut.edu.co

(b) Graduado en Ciencias Sociales de la Universidad del Tolima, Especialista en Marketing y Negocios Internacionales de Universidad de Ibagué, Candidato a Magíster Scientiae en Geografía de la Escuela de Posgrados en Geografía de la UPTC y el IGAC. Profesor de cátedra de la Universidad del Tolima, Ibagué, Colombia. http://orcid.org/0000-0003-2291-9467. oacastanof@ ut.edu.co 


\section{R E S U M E N}

El presente artículo es un ejercicio de revisión bibliográfica y análisis temático especializado, que busca establecer los rasgos más sobresalientes de la geopolítica global contemporánea. El documento contiene inicialmente los fundamentos teóricos para caracterizar desde la geopolítica y la geoeconomía los vectores que impulsan constantemente las alteraciones del orden mundial. Igualmente, aborda temáticas como los conflictos entre estados, las áreas de tensión y conflictos asimétricos entre grupos radicales y hegemonías occidentales. Por otra parte, las transformaciones a las que está siendo sometida actualmente la globalización económica se agudizan en virtud de las estrategias que los estados y las corporaciones disponen por acceder al control y administración del territorio y los recursos estratégicos en todas las escalas y en todos los espacios geográficos. El documento es una herramienta vital para estudiantes de ciencias humanas y empresariales, ejecutivos y, en síntesis, para aquellos interesados en comprender el complejo entramado geopolítico en el marco de la globalización.

PALABRAS CLAVE: conflictos, geografía política, integración económica, política económica internacional, relaciones internacionales. 


\section{A B S T R A C T}

This article is bibliographic review and specialized thematic analysis aimed at establishing the most outstanding features of contemporary global geopolitics. The document initially contains the theoretical foundations to characterize the vectors that constantly drive the alterations of the world order from the perspective of geopolitics and geoeconomics. It also addresses issues such as conflicts between states, areas of tension, and asymmetric conflicts between radical groups and Western hegemonies. Transformations in which such conflicts are currently within the sphere of economic globalization are exacerbated by the strategies of States and corporations for the control and the administration of territories and strategic resources at all scales, and in all geographic spaces. This paper is a vital tool for students in Human and Business Sciences, executives and, in short, those interested in understanding the complex geopolitical scenario within the framework of globalization.

KEY WORDS: political geography, conflicts, international economic policy, international relations, economic integration.

\section{R E S U M O}

Este artigo é um exercício de revisão da literatura e de análise temática especializada que visa estabelecer as características mais salientes da geopolítica global contemporânea. O documento contém inicialmente os fundamentos teóricos para caracterizar, a partir da geopolítica e da geoeconomia, os vetores que impulsionam constantemente as alterações da ordem mundial. Igualmente são desenvolvidas questões como os conflitos entre Estados, áreas de estresse e conflito assimétrico entre grupos radicais e hegemonias ocidentais. Além disso, as transformações às quais a globalização econômica está submetida atualmente são exacerbadas pelas estratégias de que dispõem os Estados e as empresas para acederem ao controle e à administração do território e dos recursos estratégicos em todas as escalas e em todos os espaços geográficos. O documento é uma ferramenta vital para estudantes de ciências humanas e empresariais, administradores e, em síntese, para aquelas pessoas interessadas em compreender o complexo tecido geopolítico no âmbito da globalizaçãos.

PALAVRAS-CHAVE: conflitos, geografia política, integração económica, política econômica internacional, relações internacionais. 


\section{N T R O D U C C I Ó N}

El objetivo del presente artículo es realizar una revisión teórica sobre geopolítica y caracterizar la conformación del orden geopolítico global de la actualidad de acuerdo con los factores políticos, económicos y socioculturales que intervienen en la configuración de dicho orden. Los autores presentan una revisión documental que se propone ser recibida como una herramienta administrativa para entender el entorno global actual incierto y volátil, y como un instrumento de estudio para los interesados en adquirir elementos de juicio afinados para comprender la multiplicidad de factores e intereses que intervienen en la configuración del devenir político y económico de Colombia en el escenario de la globalización.

La metodología utilizada es la revisión bibliográfica descriptiva especializada, en la cual se revisaron, analizaron y compararon artículos elaborados por especialistas en temas geopolíticos, así como la consulta de bases de datos reconocidas y acreditadas en el contexto mundial; esta estuvo enmarcada en un ejercicio hermenéutico de trabajo documental. Adicionalmente, se complementa el análisis con aportes cuantitativos que contribuyen a justificar las reflexiones y lecturas que surjan como resultado del desarrollo metodológico y que permitan tanto generar propuestas como profundizar en nuevos temas de estudio. 


\section{Precisiones teóricas preliminares: geografía política y geopolítica}

Ha pasado un poco más de un siglo desde que Friedrich Ratzel, el prolífico pensador alemán, fundó la geografía política en una serie de obras en las que el hombre y la población eran el vértice de sus preocupaciones teóricas en torno al desarrollo de las condiciones geográficas de los estados. Ratzel, en su célebre ensayo 1896, Las leyes del crecimiento espacial de los Estados (2011, p. 136), así como en su publicación "Geografía Política", de 1897, enuncia y modela los objetos de estudio de esta disciplina, y según sugieren algunos expertos, de la geografía humana en general.

A Ratzel también se le atribuye la fundación de la geopolítica como una subdisciplina subsidiaria de la geografía política. Sin embargo, la geopolítica tendría un gran alcance dado que se constituyó como una herramienta sumamente útil tanto para describir y establecer los atributos físicos y humanos de un Estado, esto es, un espacio organizado políticamente. Sin entrar en el debate sobre el carácter determinista de la disciplina en su primera etapa, hemos de afirmar que los eventos históricos relacionados con las dos guerras mundiales hicieron que la geopolítica tuviera inusitada resonancia entre los altos mandos políticos y militares de los estados protagonistas en dichas contiendas, en particular, en la Segunda Guerra Mundial.

La geopolítica en estas circunstancias fue instrumentalizada por el poder de los regímenes de aquella época. En la literatura especializada sobre el tema se ha podido establecer que la geopolítica (sobre todo la escuela alemana) se sometió al ostracismo tras el fin de la Segunda Guerra tras juzgársele en los circuitos de pensamiento anglosajones como un discurso que contribuyó al expansionismo de los regímenes totalitaristas, especialmente del nacional socialista alemán.

Afortunadamente, hoy en día esta relación ideológica parece haber sido superada tras los procesos de reformulación teóricometodológica de la geografía política a partir de la década de los 80 del siglo pasado. No obstante, el peso del Estado como objeto de estudio central dentro de la geopolítica clásica es uno de los legados de los pioneros de la disciplina, principalmente 
Ratzel. Jean Gottman, quien tuvo el acierto de "redescubrir" el potencial explicativo de la obra del pensador alemán, afirmó en su momento que la geografía política clásica es de hecho una geografía del Estado que había que rebasar proponiendo una problemática relacional en la cual la clave es el poder. En cualquier relación circula el poder, que no es poseído ni adquirido, sino pura y simplemente ejercido (Raffestin, 2011, p. 10). Este breve contexto histórico sirve para introducir al lector en los conceptos más relevantes de estas disciplinas, sobre los cuales gravitan la descripción y el análisis de los factores que determinan la configuración del nuevo panorama o el nuevo orden geopolítico.

Como lo reconociera Claude Raffestin en "por una geografía del poder" (2011, p. 18), los estudios desarrollados en la actualidad dentro de la geografía política y la geopolítica trabajan con el molde que Ratzel dejó como legado. En este sentido, según el análisis sobre el Estado, los principales principios de este son los recursos, la población y el territorio. Por supuesto, estas cuestiones se han ampliado en sintonía con el desarrollo del mundo contemporáneo, y se han introducido nuevas temáticas que están ocupando la agenda de los gobiernos y estudiosos sobre la materia.

Conviene entonces plantear las definiciones de geografía política y geopolítica con la idea subyacente de que son dos disciplinas diferenciadas. Si bien guardan una estrecha relación, es necesario subrayar que la escala de trabajo es un primer elemento que las diferencia. Ratzel, en un primer momento, plantea que la geografía política es el estudio del Estado y su dimensión territorial, es decir, para esta disciplina, el Estado es una porción de la superficie terrestre donde tienen lugar diferentes actividades humanas de carácter vital, las cuales guardan una relación simbiótica con el suelo (espacio vital) y sus condiciones geográficas.

Autores como André Sanguin (1981), Paul Claval (1978) y el mismo Raffestin (1980) han introducido nuevos elementos asociados a las relaciones de poder que se desarrollan en el marco del Estado y su territorio, así como los conflictos políticos que generan transformaciones territoriales, tal como sucedió a lo largo del siglo XX. En la misma línea de pensamiento se inscribe la definición planteada por Yves Lacoste, para quien: 
El término "geopolítica", utilizado en nuestros días de múltiples maneras, designa en la práctica todo lo relacionado con las rivalidades por el poder o la influencia sobre determinados territorios y sus poblaciones: rivalidades entre poderes políticos de todo tipo -no solamente entre Estados, sino también entre movimientos políticos o grupos armados más o menos clandestinos-, y rivalidades por el control o la dominación de territorios de mayor o menor extensión. Los razonamientos geopolíticos ayudan a comprender mejor las causas de tal o cual conflicto, dentro de un país o entre estados, pero también a proyectar los que pueden tener lugar, por tanto, las consecuencias de aquellas luchas en países más o menos alejados y algunas veces también en otras partes del mundo (Lacoste, 2009, p. 8).

En ese sentido, la geopolítica es la disciplina que analiza y explica la relación entre el espacio, el territorio y las decisiones políticas. Las decisiones políticas conciernen naturalmente al Estado, pero también a otros actores políticos minoritarios en escalas más pequeñas, que pueden generar transformaciones territoriales al interior de un país (p. ej. las organizaciones sociales y económicas, los movimientos separatistas e incluso los individuos con capacidad económica e influencia para controlar la organización económica de un territorio, etc.). Para Lacoste, las escalas espaciales o magnitudes de escala en la geografía política son fundamentalmente tres:

1. Escala global: relacionada con el análisis de problemas y fenómenos que involucran a varios estados.

2. Escala estatal o nacional: el análisis de problemáticas al interior de un Estado.

3. Escala local/regional: una escala más detallada de trabajo tiene que ver con el análisis de problemáticas que se dan en ciudades o municipios de un Estado. Los resultados de dichos procesos microescalares pueden influir en la configuración territorial en el contexto nacional.

La geopolítica es un área rica de trabajo y es altamente interdisciplinar, guarda una profunda relación con la ciencia 
política, la historia, la sociología, la geoestrategia, así como con otras áreas de la geografía social, tales como la geografía agraria, la geografía económica y la geografía urbana. Esta riqueza conceptual y metodológica hace que el perfil de análisis geopolítico sea mucho más completo e integrador. Tal vez esta virtud pueda explicar el hecho que durante gran parte del siglo XX estuvo muy activa como herramienta de toma de decisiones por parte de los gobiernos, al tiempo que permaneció proscrita como disciplina por las causas mencionadas al principio.

La geopolítica por su parte también ha experimentado una reorientación teórico-metodológica, en la cual se destacan los esfuerzos de los países anglosajones, que dieron como resultado el situar en un lugar privilegiado a la disciplina, sobre todo cuando los Estados Unidos se valieron de dicha herramienta para diseñar su estrategia internacional a finales de la década de los 80 del siglo XX. En términos epistemológicos la disciplina no fue ajena a los debates que se dieron a partir de la segunda mitad del siglo XX en el seno del pensamiento geográfico, los cuales buscaban dotar de un sistema conceptual propio a la geografía humana en general. Paul Claval ilustra esta pretensión:

El espacio estudiado por "la nueva geografía" no está formado, únicamente, por elementos físicos o naturales. Está poblado por personas que establecen lazos entre sí. Estas relaciones, cuando duran, dan lugar a redes. Estas son, en primer lugar, realidades sociales, puesto que unen a negociadores unidos por sus asuntos, profesionales que necesitan consultarse periódicamente, o parejas de vendedores y compradores, prestadores de servicios y clientes. Estas redes también son realidades materiales, puesto que los desplazamientos de personas e intercambios de bienes se llevan a cabo gracias a las vías de comunicación; las informaciones, noticias y órdenes circulan por redes de telecomunicación (Claval, 1999, p. 28).

La geopolítica se constituye en una disciplina privilegiada para analizar los factores que afectan la estabilidad política de los estados y de una región. Entre los objetos de estudio más destacados hoy en día y a futuro podemos mencionar: 
- Seguridad y soberanía nacional.

- Relaciones internacionales.

- Conflictos interestatales.

- Megaproyectos de infraestructura.

- Integración económica.

La geopolítica, para un Estado con intereses en estas áreas, según Ballesteros (2014), “[...] sirve para analizar el escenario en que debe desarrollar su campo de acción. Su objetivo es facilitar la toma de las decisiones políticas, proporcionando el conjunto de informaciones que faciliten el trabajo de los decisores" (p. 3). Esas informaciones pasan por un conjunto de variables que deben apuntar a un conocimiento detallado del territorio en cuanto medio físico y soporte de las relaciones sociales y actividades vitales para los habitantes de un país, por supuesto las variables relacionadas con el poder y la estructura del Gobierno son cruciales para entender el contexto.

Definimos entonces la geopolítica como la disciplina que estudia las relaciones de poder en la escala estatal e interestatal, y las transformaciones espaciales producidas en ese escenario. Este estudio implica el análisis sistemático de las condiciones geográficas de la superficie terrestre y marítima controlada por un Estado, así como de condiciones sociopolíticas, económicas y militares.

La geopolítica, de acuerdo con Dallanegra (2008, p. 16):

es un objeto de estudio dinámico e interdisciplinario que requiere del marco teórico que le ofrecen las ciencias políticas, las relaciones internacionales, la geografía, y otras ciencias como la economía y la historia. Las relaciones internacionales contribuyen con el concepto de poder, desempeño, interrelación y configuración sistémica. La geografía hace lo propio con la idea de espacio vital; la economía con la noción de riqueza y recursos y la historia con la concepción de evolución y dinámica.

En el marco de las relaciones internacionales, los expertos han desarrollado métodos de análisis que permiten estudiar los cambios, riesgos y amenazas a la estabilidad de los estados en el marco de la globalización. Las regiones geopolíticas sirven para encarar esta cuestión. El profesor Miguel Ángel Ballesteros las define como 
Un conjunto de Estados que, desde el punto de vista del estudio geopolítico, presenta un cierto carácter de unidad o de integración, bien sea física, humana, económica, política, etc. que hace que las acciones en cada una de sus partes estén estrechamente relacionadas con los efectos en las otras (2013, p. 13).

En ese sentido, vale la pena aclarar que las relaciones entre los Estados son, la mayoría de las veces, asimétricas, y están dadas por el nivel de desarrollo de las fuerzas económicas, políticas y militares de cada uno. Immanuel Wallerstein (1974, p. 347) ya lo había planteado a través de su teoría del sistema-mundo, en la cual se establece que la economía mundial presenta interconexiones y jerarquías dentro de un esquema de centro y periferia. Para caracterizar esta condición predominante del panorama actual es útil la noción de hegemonía en el sentido que Eric Hobsbawm la ha planteado.

En sus análisis sobre el imperialismo y la conformación del orden mundial a lo largo del siglo XIX y XX, Hobsbawm (1999, p. 12) denomina estados hegemónicos a aquellos que concentran una superioridad económica, política, científica y militar que les permite intervenir (con arreglo a sus intereses) activamente en las decisiones de otros estados más débiles y dependientes.

Esto fue un rasgo fundamental de la geopolítica global desde el siglo XIX. En un ejercicio comparativo entre Gran Bretaña y Estados Unidos, el historiador inglés desarrolla un análisis de los ejercicios hegemónicos de las dos potencias. En primer lugar, afirma que "la clara superioridad técnica, económica y, por lo tanto, militar del centro sobre la periferia se incrementó enormemente en el siglo XIX, gracias a las denominadas 'herramientas del imperio" (1997, p. 1), subrayando que, para el caso norteamericano, su hegemonía depende altamente de la variable militar. A la luz de los cambios introducidos por la administración Obama en la política internacional, habría que establecer si esta dependencia se mantiene.

Modernamente las áreas de estudio de la geopolítica se han ampliado. Una de ellas, de mucha importancia es la geoeconomía, también el análisis ambiental, minerales estratégicos, la defensa de las multinacionales y otras que más adelante tendrán espacio en el presente artículo 


\section{Análisis de factores estratégicos. Geopolítica: conflictos y focos de tensión en la escala global}

Al ser el territorio y los recursos variables fundamentales de la organización política estatal, la lucha por el control, defensa y apropiación del suelo (entendido como la superficie terrestre y marítima delimitada a través de fronteras científicas) se constituye como un compromiso del cual depende la estabilidad sociopolítica interna de los estados, así como su influencia en el concierto geopolítico en la escala regional y global.

Durante el siglo XX, las contiendas mundiales fueron el punto culminante de diferentes conflictos sucedidos a lo largo del siglo XIX. La expansión territorial de diferentes potencias europeas se libró en el propio continente, así como en otros espacios geográficos en una etapa que historiadores como Eric Hobsbawm (1998) han denominado el imperialismo. La permanente reconfiguración del mapa político mundial desde las coyunturas de las dos posguerras y el desmantelamiento del bloque socialista liderado por la ex URSS en la segunda mitad del siglo XX nos ha hecho testigos del surgimiento de nuevos estados y la reconfiguración de otros tantos. Por ejemplo, al finalizar la gran guerra en 1918 y tras la firma del acuerdo Sykes-Picot, los antiguos imperios europeos y sus dominios de ultramar se fragmentan para dar paso a nuevos estados como se puede observar en la figura 1 sobre Europa antes y después de la paz de París:

Los conflictos ${ }^{1}$, de acuerdo con Ricardo Méndez, tuvieron a Europa como protagonista durante gran parte del siglo XX. Los continuos cambios en su mapa como unidad política continental han caracterizado su larga historia. Esos cambios tienen que ver también con dos fuerzas en disputa: por un lado, los proyectos integracionistas, y, por el otro, la permanente fragmentación espacial que se ha traducido en los esfuerzos separatistas de diversos

1 Según Ricardo Méndez, en su análisis sobre la geografía de los nuevos conflictos, puede definirse el conflicto, teniendo en cuenta que "toda sociedad genera contradicciones y enfrentamientos, tanto en su interior como con otras, surgidos cuando dos o más individuos, grupos u organizaciones persiguen similares objetivos, incompatibles entre sí o que, al menos, se perciben como tales" (2011, p. 4). Este planteamiento puede aplicarse al análisis de los conflictos interestatales, uno de los principales objetos de estudio de la disciplina. 

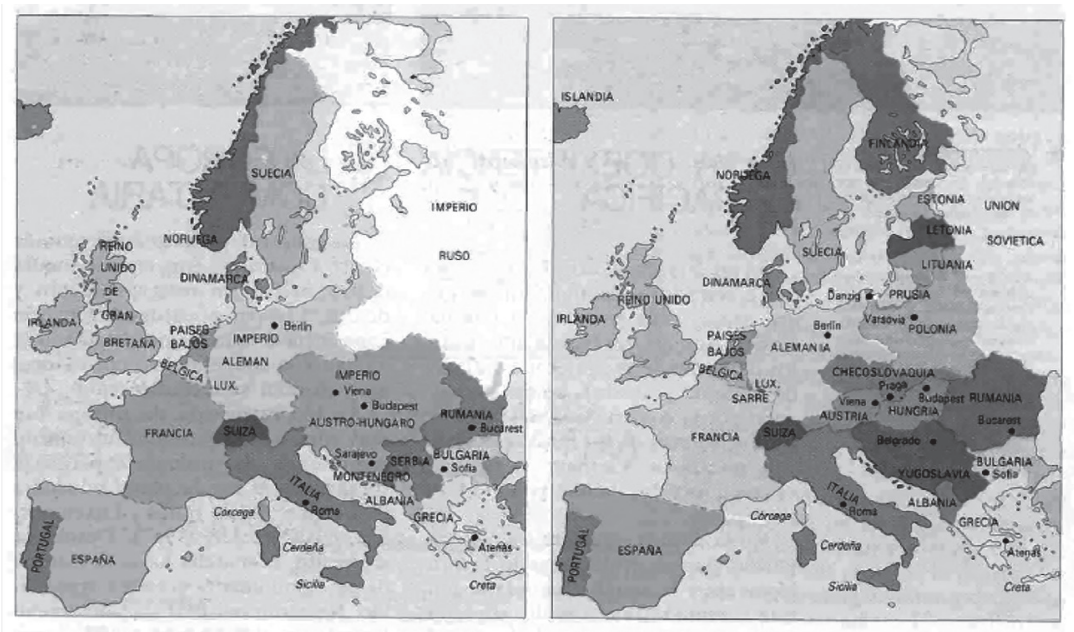

Figura 1. Europa: antes y después de la Primera Guerra Mundial.

Fuente: UNLP.

movimientos nacionalistas culturalmente diferenciados que no pocas veces se han concretado. La división en varios estados de los territorios que antes conformaban Yugoslavia en la región de los Balcanes, sirve para explicar este planteamiento.

Dado este escenario, el mundo pasó de tener aproximadamente 60 estados nacionales antes de 1945 a contar con aproximadamente 200 entidades estatales después de ese año. Las transformaciones espaciales derivadas de los conflictos territoriales a escala interestatal ocupan la agenda geopolítica de los gobiernos y los estudiosos del tema.

La conflictividad asociada a las confrontaciones por el territorio sigue ocupando un lugar central en la geopolítica del siglo XXI; sin embargo, dos referentes han introducido nuevos actores y variables que agudizan y hacen más complejo comprender los hechos en tal escenario.

Varios expertos han planteado que los acontecimientos del World Trade Center generan cambios sustanciales en la geopolítica global y las estrategias internacionales norteamericanas. Al respecto el profesor Hernández Alvarado (2002, p. 17) dice:

A partir de los atentados del 11 de septiembre del año pasado, la principal tendencia en el ámbito geopolítico apunta a la 
configuración de un mundo en conflicto [...] los EE. UU. están configurando un mundo conflictivo donde la "disuasión convencional" ha venido a sustituir a la "disuasión nuclear" de la Guerra Fría, que contrasta con las tendencias de la administración Clinton. El concepto de seguridad nacional e internacional se convierte en el valor fundamental y en el parámetro para medir el comportamiento de los gobiernos y de los países.

Lacoste, también en la misma línea, afirma sobre el surgimiento de una geopolítica mucho más volátil:

Las causas son complejas y resultan, en gran parte, del desarrollo durante más de treinta años de consecuencias geopolíticas sobrevenidas en Medio Oriente. Pero las causas más directas del suceso, las cuales siguieron a la Guerra del Golfo de 1991 tras la invasión de Kuwait, se sitúan más precisamente en Arabia Saudita -aun cuando es antigua aliada de Estados Unidos-, al seno de dirigentes medianos riquísimos, a razón de rivalidades políticas entre personajes cercanos al soberano y un millonario islamita ligado tiempo atrás a los servicios secretos norteamericanos respecto a los asuntos de Afganistán: Osama Bin Laden, en este caso. El ataque del 11 de septiembre es siempre percibido por los norteamericanos como el equivalente del ataque japonés a Pearl Harbor en 1941 (2009, p. 8).

En ese sentido, los conflictos y tensiones del siglo XXI hacen al yihadismo musulmán un actor poderoso, con una notable capacidad bélica y de desestabilización global, pues usan al terrorismo como arma de agresión contra los gobiernos occidentales y EE. UU., especialmente. Así mismo, el control y la influencia sobre la región de medio oriente serán neurálgicos para los intereses de Washington y su pretensión de conducir el orden geopolítico global y conservar su posición dominante como Estado hegemónico. Más adelante se analizará este punto con mayor detalle.

El segundo referente lo constituye un evento asociado al descrito anteriormente. La intervención de las hegemonías occidentales y de EE. UU, particularmente tras su invasión a Irak en el 2003 y su partida del país en el 2011, ha intensificado y agravado 
el conflicto territorial entre sunitas y chiitas (las dos grandes ramas del islam), pues el interés de Washington es reconfigurar funcionalmente a su agenda, la región de oriente medio y el norte de África. La estabilización de estos conflictos no parecía ser su prioridad hasta la firma de los acuerdos de Lausana entre Washington y el nuevo gobierno iraní en el 2015, lo cual hace parte de una nueva estrategia norteamericana por ganar nuevos aliados y debilitar la influencia de Rusia en la región tras las transformaciones geopolíticas de la primavera árabe.
El perfil de los conflictos actuales es confuso y discontinuo, como lo señala Ricardo Méndez (2011). Estos tienen lógicas espaciales distintas a los conflictos que marcaron el siglo XX. La geopolítica global contemporánea guarda rasgos relacionados con el caos y los altos niveles de riesgo que presentan determinados países en virtud de sus niveles de conflictividad e inestabilidad político- económica. El profesor Méndez explica esta nueva condición con gran claridad: En este escenario, la nueva fisonomía de la guerra, la multiplicación de actores implicados en la misma, el desplazamiento de las regiones más afectadas por la violencia armada, o la aparición de nuevas causas de conflicto bélico que se suman a otras de larga tradición justifican la necesaria renovación de una de las temáticas clásicas de los estudios geopolíticos (2011, p. 21).

Es necesario en este punto mencionar la importancia que la economía de la guerra juega en este complejo ajedrez geopolítico. Con la consolidación de la globalización económica también se consolida la lucha entre los estados hegemónicos por controlar el mercado de armamento y de los servicios de seguridad y defensa. Según el Instituto Internacional de Estudios para la Paz de Estocolmo (SIPRI), la oferta mundial de armas se ha incrementado en un $14 \%$ en los últimos cuatro años, conservando la tendencia al alza iniciada a partir del 2002.

Vale la pena observar el comportamiento del mercado mundial de armamento para el periodo 2011-2015, y precisar la participación de los diferentes países productores y compradores en el mercado. India y Arabia Saudí se ubican como los principales importadores. El caso de la India es llamativo, pues no ha consolidado una industria 


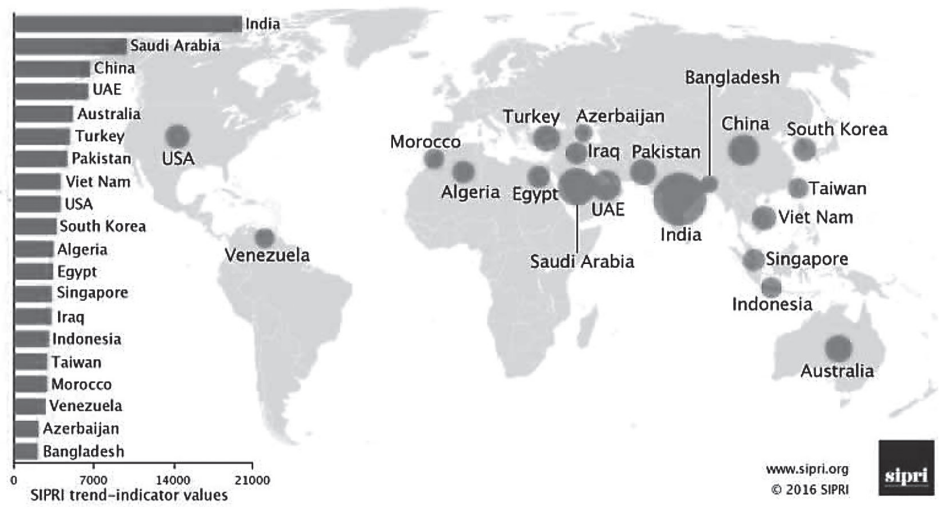

Figura 2. The 20 largest arms importers 2011-2015

Fuente: SIPRI recuperado de

https://www.sipri.org/research/armament-and-disarmament/armstransfers-and-military-spending/international-arms-transfers

armamentística propia y sofisticada, lo que se traduce en niveles de calidad e innovación inferiores a los de China, uno de sus vecinos en la región.

Así como Asia y Medio Oriente lideran las importaciones del mercado de armamento, por el lado de la producción, el Instituto argumenta, que Estados Unidos se mantiene en el top del mercado de la exportación de armamento. Según el SIPRI:

Con el 33\% del total de las exportaciones de armas, Estados Unidos fue el mayor exportador de armas en el periodo 20112015. Sus exportaciones de grandes armas crecieron un $27 \%$ en comparación con el periodo 2006-2010. Las exportaciones de grandes armas de Rusia crecieron un 28\% entre 2006-2010 y 2011-2015, y el país contabilizó el 25\% del total de exportaciones en el último periodo de cinco años. Sin embargo, en 2014 y en 2015, las exportaciones rusas volvieron a los niveles anuales más bajos observados en el periodo 2006-2010 (2016, p.17).

China, por su parte, desplazó a Francia como segundo productor de armas, y aumentó en un $88 \%$ su gasto militar (la producción y exportación), mientras que Alemania, uno de los tradicionales líderes del mercado, redujo su participación a la mitad. 
La situación de China contrasta con la de India, siendo países vecinos que incrementaron su participación como importadores y exportadores, en gran parte debido a su ascenso como potencias globales y líderes de un polo hegemónico integrado también por Rusia, y a la amenaza latente de inestabilidad que proviene tanto de Afganistán como Pakistán. Los conflictos y tensiones regionales se mantienen, y esto impulsa a los países a buscar una mejor posición para enfrentar, ya sea por la vía militar, ya sea por la política, las amenazas que se ciernen a diario sobre la seguridad estatal y global.

\section{Un nuevo orden mundial: el significado del término}

En su sentido más amplio, es indispensable entender la denominación nuevo orden mundial, según Chomsky (2007, p. 62), como una nueva época de la historia mundial, como una oportunidad de cambios en el equilibrio de poderes o como un nuevo periodo de estabilidad social, política y económica y, para el grueso de habitantes significará una nueva etapa de paz y tranquilidad.

El avance de la globalización económica permite observar la dinámica de este nuevo orden en la escala interestatal. Para Manuel Castells, la globalización “[...] es un proceso esencialmente económico que implica la interconexión permanente de los procesos productivos en todas las escalas y en tiempo real" (2000, p. 45). En esa constante red las distancias y el tiempo son fricciones que las tecnologías de la información y la comunicación superan para reforzar cada vez más las relaciones entre los estados, que indudablemente guardan un carácter asimétrico.

En el mismo orden de ideas, la intensificación y agudización de los procesos de globalización en el mundo entero presionan a los países a buscar mayores niveles de productividad y competitividad. En este caso, los estados aportan y, a la vez, presionan permanentemente a sus organizaciones comerciales a que alcancen efectividad en estas dos variables tan cruciales en el desarrollo de los países en el caso de países pequeños; a sus multilatinas, para los países en vía de desarrollo, y multinacionales, para los países con mayores niveles de desarrollo. 
Este fenómeno ha traído para todas las naciones una desbocada carrera por la conquista de los grandes clientes internacionales, y solo aquellos países con tradición, experticia, conocimiento de los mercados, preparación en marketing, gran oferta de bienes y servicios y cobertura geográfica, aquellos que cumplen con las anteriores condiciones, tendrán bajo su dominio la demanda mundial. Esto se traduce en un nuevo orden del comercio global, destacando que cada día adquiere mayor preponderancia el comercio y menos interés por las disputas entre países, porque quienes poseen mayores y mejores mercados tienen a su vez todas las posibilidades de imponer sanciones comerciales, estrategia de presión que condena al ostracismo a naciones como Cuba, o situaciones desestabilizadoras como la actual que vive Rusia, que, en el mejor de los casos, se superan como en Irán.

El orden geopolítico mundial en el decenio de los 90 estuvo precedido por el debilitamiento y posterior caída del modelo económico de URSS, que se encontraba en aguda crisis desde 1985 y se desmembró en diversos países, lo que coincidió con la caída del muro de Berlín; el surgimiento del glásnost y la perestroika en la nueva Rusia; la consolidación industrial y exportadora de los tigres asiáticos (Corea del Sur, Taiwán, Singapur y Hong Kong), y el final de la guerra fría.

Al mismo tiempo en occidente ocurre un fortalecimiento del modelo desarrollista neoliberal liderado por EE. UU. y Gran Bretaña, y secundado por las instituciones de gobernanza global (ONU, FMI, BM y OTAN) e instituciones comerciales como multinacionales, transnacionales y el sector financiero global; este modelo se complementó por el referente de una cultura occidental a transferir a diversas naciones del globo.

Sin embargo, es de destacar que la prolongada guerra fría que hubo hasta los 90 generó una intensa polarización entre EE. UU. y Rusia; en la actualidad no hay con claridad en el nuevo siglo un país líder. Podría decirse que actualmente el mundo es apolar, ya que no existe una hegemonía definida; en el mejor de los casos, la multipolaridad emerge de manos de tres posibles líderes globales: EE. UU. China y la Unión Europea. Además, un nuevo actor emerge con fuerza de las cenizas: Rusia, antigua URSS.

Se ha realizado un análisis en profundidad desde la geopolítica acerca del nuevo orden mundial durante los primeros dos decenios 
del nuevo siglo. El panorama luego de este examen requiere obligatoriamente detallar un arco argumental temático relacionado con la identificación de nuevos riesgos globales en la seguridad, la gobernanza, la economía y el comercio global. A su vez, este estudio presenta tensiones geopolíticas que presentan un mundo muy diferente al que existía antes: establecer el nuevo papel de EE. UU. en el gobierno mundial; conocer si el alto al fuego en Siria auspiciado por EE. UU. y Rusia detendrá la mortandad, destrucción y la migración desaforada; analizar las repercusiones de la salida del Reino Unido de la Unión Europea; estudiar si el mar meridional de China agudizará las tensiones entre China y los países aledaños, y si realmente ocurrirá una crisis de izquierda en los gobiernos latinoamericanos con el retorno de la derecha.

\section{Tipos de riesgos globales}

Los riesgos son eventos que generan incertidumbre porque son imprevisibles, máxime cuando también lo son sus posibles impactos, y solo hacen mantener en vilo a las comunidades y socavar la confianza y credibilidad en los gobiernos.

La Global Risk Perception Survey del 2014 que hizo el Foro Económico Mundial identifica los diez principales riesgos por su probabilidad:

- Conflictos entre estados.

- Deficiencias de la gobernanza nacional.

- Colapso o crisis del Estado-nación.

- Desempleo o subempleo.

- Catástrofes naturales.

- Falta de adaptación al cambio climático.

- Crisis del agua.

- Ataques cibernéticos.

El Foro identifica otros diez principales riesgos por su impacto:

- Crisis del agua.

- Pandemias.

- Armas de destrucción masiva. 
- Conflictos entre estados.

- Falta de adaptación al cambio climático.

- Crisis de los precios de la energía.

- Crisis fiscales.

- Pérdida de biodiversidad.

\section{Geoeconomía: en defensa de los recursos}

El nuevo orden mundial también reconfigura nuevos elementos disciplinares de análisis; muy importantes son la geoeconomía y la geoestrategia. Esto significa que el ámbito analítico de la geopolítica se amplía acorde a la expansión y agudización de las problemáticas y la contundencia de respuestas a las lecturas del entorno internacional. Por esto, requiere de nuevas disciplinas.

Eduardo Olier (2011, p. 17), en su artículo "la geopolítica de la economía global", la define como una nueva ciencia encargada del análisis de las estrategias de orden económico y comercial decididas por los estados con base en sus intereses, con el fin de proteger sus economías en el contexto económico global o dotarse de las tecnologías o productos que consideran estratégicos para su supervivencia o desarrollo futuro. Para Luttwak, "[...] la geoeconomía es el mantenimiento de la antigua rivalidad existente entre las naciones utilizando medios económicos en lugar de bélicos" (1990, p. 17).

La geoeconomía aporta a la geopolítica análisis internacionales integrales desde la ciencia económica, los negocios globales, el mercadeo internacional y los recursos económicos. Esto está complementado con nuevos escenarios, interpretaciones y soluciones en un ambiente globalizador que no permite las dilaciones de los gobiernos de izquierda o de derecha; por el contrario, fusiona regiones completas y las acomoda de acuerdo a sus intereses en bloques de comercio integrado, lo que favorece la expansión del consumismo y la hiperconectividad mundial, y ningún país desea para sus habitantes el ostracismo y el consecuente atraso

En el marco de las lecturas geopolíticas, la geoeconomía apunta a estudiar e identificar los mejores escenarios de negocios en el mundo; la interacción con multinacionales y cadenas globales de 
producción, abastecimiento y distribución; el reconocimiento de los actores políticos en los países de destino que pudiesen apoyar los nuevos emprendimientos y diseñar las mejores estrategias de marketing para mejorar la competitividad de los negocios en los mercados de referencia. Es este el análisis a un avasallador contexto comercial global catapultado por los estados y por los efectos de la globalización económica rampante que desplaza lentamente a la geopolítica. En este escenario se prefiere y defienden las estrategias de las multinacionales y los tratados de libre comercio, y en menor grado las estrategias político-militares de los estados.

Por su parte, la geoestrategia también amplía su radio de acción como disciplina de la geopolítica. Mientras que esta última se encarga de analizar las causas y consecuencias de las relaciones políticas, sociales, culturales y económicas entre los estados y en diferentes escenarios, la geoestrategia estudia en diferentes contextos el desarrollo de directrices de alta productividad y competitividad para afirmar la seguridad económica mediante el impulso a organizaciones multinacionales rentables que posicionen sus productos y servicios en muchos países del mundo. Se convierte el comercio en un arma mortífera en manos de nuevos actores como las multinacionales y las cadenas globales de retailers.

En este orden de ideas, los aspectos relevantes que se deben tratar desde la geoeconomía son los siguientes:

1. El decrecimiento del comercio mundial.

2. Las fuertes expansiones comerciales de las multinacionales.

3. El fortalecimiento de las cadenas de suministro, producción y distribución globales, en las cuales participan procesos de automatización y robots, que posibilitan nuevos escenarios de productividad.

4. La expansión de la cobertura de los tratados de libre comercio.

5. La importancia de la tecnología de comunicaciones en la interacción entre compradores y vendedores globales por efecto de lo que se ha denominado la cuarta revolución industrial y la hiperconectividad, cuya evolución les permite a los ciudadanos del mundo tener acceso a información de manera veloz, continua y universal.

6. El análisis de aspectos negativos como los bloqueos económicos y sanciones económicas. 
Respecto al decrecimiento del comercio mundial es importante tener en cuenta las siguientes cifras. Para el 2014, según la Organización Mundial del Comercio, el crecimiento fue de apenas $2,8 \%$, superando levemente el 2,4\% del 2013. Sin embargo, el valor de las exportaciones mundiales de bienes solo creció un $0,7 \%$ en el 2014, mientras que el de las de servicios lo hicieron al $4 \%$.

En el 2015, el volumen del comercio de mercancías en el mundo aumentó solamente un 2,6\%. Diferentes causas son atribuibles al fenómeno, como el crecimiento sostenido, pero lento, de la economía norteamericana, la abrupta caída del precio de las commodities en las economías suramericanas, la desaceleración de Europa y China, y el dramático decremento de las economías Europa central y del este, en buena parte por las sanciones económicas al principal motor regional, para este caso, Rusia.

Situación contraria sucede con las multinacionales, organizaciones con gran capacidad de adaptabilidad al entorno global, que poseen cultura y visión global de los negocios. Se enfocan en la universalización de los productos, en su core business y en la rentabilidad por efectos del uso de economías de escala y de investigación, lo que conlleva a trasladar menores costos en forma de precios bajos al comprador final, con fuertes inversiones en investigación y desarrollo; esto a su vez ha repercutido en mayores ventas y ampliación de su cobertura mundial. Al respecto, la revista Fortune 500 presenta el ranking de organizaciones mundiales más grandes y valiosas para los años 2005 a 2015.

La figura 3 muestra un marcado dominio de las corporaciones del sector petrolero tanto en el 2005 como en el 2015, así como de las empresas del sector automotriz. Esta tendencia se conservará en el mediano plazo, pues es claro que el poder de dichas compañías retrasa el tránsito hacia las fuentes de energía limpia; asimismo, la llegada al poder de Donald Trump y su política sobre cambio climático le ha otorgado más poder y beneficios al sector de hidrocarburos, ya que ha desregulado las actividades de extracción mediante fracturación hidráulica.

Adicionalmente, en el siguiente figura de las mayores economías del mundo también se puede analizar desde la arista geoeconómica: el PIB muestra que hay paridad de compra para las principales potencias económicas China y EE. UU. en donde se pueden apreciar las magnitudes de los indicadores respecto de 


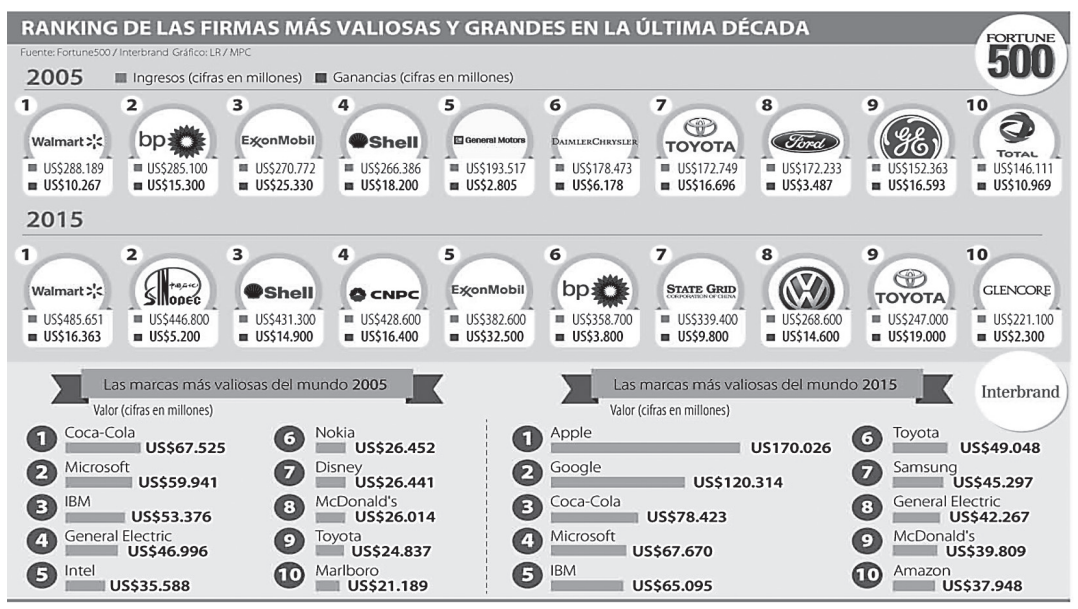

Figura 3. Ranking de las firmas más valiosas y grandes en la última década.

Fuente: Fortune, recuperado de http://fortune.com/global500/

los demás países y su jerarquía dominante en el conjunto de países comercialmente más desarrollados en el ámbito global.

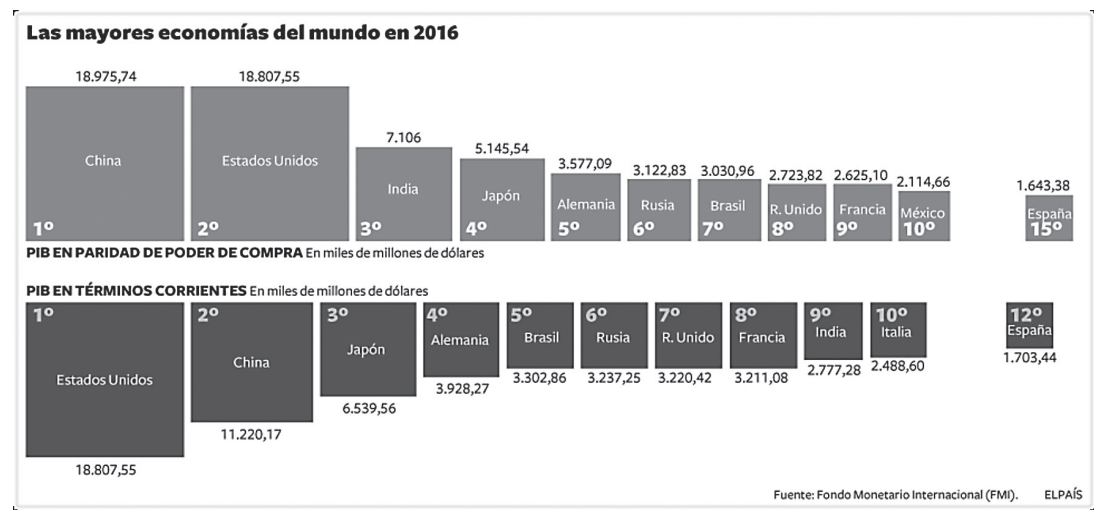

Figura 4. Las mayores economías del mundo.

Fuente: FMI.

Complementariamente, de la misma manera como se incrementa la demanda mundial por productos de buena calidad y de bajo precio, intervienen las cadenas globales de proveedores de insumos, de producción de bienes universales y de distribución de artículos finales. Las cadenas apoyan la existencia de las multinacionales, en especial, las cadenas globales de producción, 
como la fábrica de América del Norte, la fábrica de Europa y la fábrica de Asia, y el propósito de los países desarrollados.

En cuanto a los tratados entre países que persiguen objetivos comunes, existen unos con propósitos políticos, y otros, comerciales. Estos últimos, que interesan a la geoeconomía, son denominados tratados de libre comercio, los cuales han servido de base a los procesos globalizadores en el mundo, están avalados por el derecho internacional, y su objetivo es diseñar y desarrollar políticas comerciales que faciliten la movilización de mercancías entre los países miembros como la reducción de los aranceles.

En la primera mitad del siglo pasado la mayor parte de los países tuvieron modelos económicos proteccionistas, pues el pensamiento de sus líderes era que cada país debía ser autosuficiente si quería crecer, y se impusieron los procesos de sustitución de importaciones y todo lo que se producía era consumido por la población nacional. A mediados del siglo pasado, 23 países fundaron el acuerdo general de aranceles y comercio (GATT), el cual para 1990 cubría alrededor de 100 países; su objetivo inicial era reducir los aranceles, eliminar prácticas comerciales restrictivas al comercio internacional y promover la cooperación y comercialización de mercancías entre países miembros.

Complementariamente a los anteriores procesos impulsadores del comercio internacional, surgió en 1995 la Organización Mundial del Comercio (OMC), actualmente con 162 países miembros. Esta tiene las siguientes funciones²:

- Administrar los acuerdos comerciales de la OMC.

- Servir de foro para negociaciones comerciales.

- Resolver las diferencias comerciales.

- Supervisar las políticas comerciales nacionales.

- Proveer asistencia técnica y cursos de formación para los países en desarrollo.

- Diseñar procesos de cooperación con otras organizaciones internacionales.

2 Con base en el siguiente link: https://www.wto.org/spanish/thewto_s/whatis_s/ whatis_s.htm 
Mientras que en el 2002 existían en el mundo alrededor de 70 TLC, a inicios del 2014, llegaban a los 550. La expansión estuvo motivada especialmente por las cadenas globales de valor, la universalización de los productos, los procesos de globalización rampante, la búsqueda constante de mayores niveles de productividad y la instantaneidad de la información que acercan cada vez más a proveedores, distribuidores y compradores globales

\section{Nueva geopolítica internacional de Estados Unidos}

Aunque no exista todavía un país hegemónico claramente reconocido, es evidente que EE. UU. continuará siendo líder dentro de los países, con autoridad y poder de persuasión y disuasión en el concierto mundial. EE. UU. siempre se caracterizó por gobiernos con mano dura, en parte con el apoyo de gobiernos amigos e instituciones de gobernanza global y poder militar, y sus aventuras bélicas tuvieron gran transcendencia en Corea, Vietnam, Panamá e Irak entre otras, lo cual generó altos costos económicos y vidas humanas; un gran costo negativo ante la opinión pública norteamericana y entre el grueso de países, y una imagen de policía del mundo, país guerrerista, invasor e imperialista.

Es indudable que se debía reducir las incidencias de la imagen negativa, lo cual se ha venido logrando a través, no de las armas, sino proyectando a sus multinacionales y cadenas globales de producción por todo el mundo. Quizás en el nuevo escenario mundial se cambian las sofisticadas armas por la expansión del comercio con bases de referencia fundamentales como lo son la agudización de los procesos de globalización, la productividad y competitividad, y la conformación de bloques comerciales, temas pertenecientes a la geoeconomía.

Por otra parte, la referida imagen negativa también se ha disminuido en los dos gobiernos del presidente Obama. Hechos como el acercamiento a Cuba luego de la ruptura de relaciones diplomáticas prolongada, el acuerdo nuclear con Irán o la coalición con Rusia, Francia, Reino Unido, Alemania y varios países más para hacer frente a ISIS hacen pensar que EE. UU. no quiere seguir liderando aventuras bélicas en ninguna parte del mundo. Mientras esto sucede, China y 
Rusia luchan por obtener un reconocimiento como líderes mundiales, aunque no lo logran, pues su alcance como hegemonías en términos geográficos es limitado, dado que históricamente han cultivado relaciones con un reducido grupo de países de Europa, Asia y África. Estas relaciones se mantuvieron fluidas en la etapa de la Guerra Fría, pero, tras el colapso del bloque socialista, se destruyeron, y EE. UU. ascendió como única potencia global.

Lo anterior implica en términos de la realpolitik para el gobierno norteamericano continuar asumiendo el compromiso con el mundo occidental y con el mundo entero. En el mismo contexto, en la recta final del doble periodo del presidente Obama, también se advierte su deseo de legar a Latinoamérica un ambiente de paz, y de tranquilidad en Argentina, Colombia y Cuba, países de la región los cuales ha visitado con el propósito de extender confianza, ayuda financiera y mejores perspectivas comerciales.

Aun con este panorama, el mundo advierte la ausencia de liderazgo y de gobernanza global, y muchos culpan del fenómeno a los gobiernos demócratas de EE. UU. El mundo padece ahora impactos irregulares de mayor fortaleza como el terrorismo con ataques suicidas impredecibles y peligrosos en cualquier país, con la expectativa de que puedan tener acceso en algún momento a armas de destrucción masiva y sean capaces de ocasionar daños mayores. Son estas las denominadas guerras asimétricas.

Adicionalmente el mundo observa con mucho cuidado una lucha rampante por recursos estratégicos de minerales, agua, petróleo y nuevas formas de energía alternativos, los cuales son cada vez más difíciles de obtener, y que garantizan la hegemonía y la capacidad de influir en el equilibrio del nuevo orden mundial.

\section{Reconfiguración geopolítica en Medio Oriente}

La situación geopolítica en Medio Oriente viene presentando un peligroso escalamiento en el que participan numerosos actores, desde pequeños grupos, denominados brigadas locales o milicias, pasando por coaliciones de naciones, hasta la vinculación en el conflicto actual de las grandes potencias militares. Por una parte, están los aliados occidentales liderados por EE. UU., Francia, Reino Unido, Canadá, como Arabia Saudita, Turquía, entre los 
más importantes, los cuales están en contra del actual presidente de Siria Bashar al-Ásad y de ISIS. El interés de EE. UU. es proteger sus amigos árabes, kurdos, rebeldes moderados, israelíes, turcos y europeos los cuales han sido atacados por células de ISIS.

Siria, país con diez millones de habitantes y liderada por Ál-Asad, fue invadida desde julio del 2014 por ISIS desde Irak, e inicialmente llegó a perder en la confrontación bélica buena parte de su territorio, alrededor del 60\%; sin embargo, paulatinamente he venido recuperándolos de la mano de la coalición formada al principio por Rusia y Francia en noviembre de 2015 y apoyada desde inicios del presente año por Estados Unidos. Desde el inicio de la invasión en Siria, han muerto 500.000 personas y se han desplazado aproximadamente cuatro millones de sirios, quienes a su vez han generado una de las peores crisis migratorias en Europa. Siria cuenta actualmente con alrededor de 90.000 militares y dos aliados importantes, Rusia e Irán. El interés del presidente sirio es conservar el poder político y militar en el país, continuar con el apoyo militar de sus actuales aliados y pactar con Estados Unidos y demás países occidentales involucrados en el conflicto un cese al fuego. Sin embargo, es de destacar que el gobierno de Ál-Asad ha estado precedido desde el 2000, año de su posesión, de una discutible imagen de gobierno represivo, torturador y asesino tanto de civiles como de opositores políticos.

Ubicado dentro de Siria, en el bastión de Ál-Asad, están las fuerzas rusas con el propósito de defender a toda costa el gobierno sirio en la base naval de Tartus, única de los rusos sobre el Mediterráneo, y en ocho puntos adicionales de concentración de efectivos rusos. Los rusos hacen ataques aéreos permanentes sobre las fuerzas de isis y posiblemente contra fuerzas rebeldes apoyadas por EE. UU., que de comprobarse podría en cualquier momento generar un enfrentamiento de mayores consecuencias.

ISIS inició actividades militares desde 1998, pero hizo su aparición en el conflicto desde el 2014. En la actualidad está ubicada en la parte norte de Siria. Inicialmente, llegó a tener más de 45.000 efectivos; sin embargo, los embates de la coalición además de territorios le han quitado alrededor de la mitad de su ejército. Actualmente solo tiene el respaldo de alrededor de 22.000 efectivos, en su mayoría yihadistas radicales, quienes empezaron siendo miembros de Al Qaeda. A la fecha esta organización ha extendido 
sus tentáculos hacia varios países vecinos y hacia otros continentes y países, incluidos Europa, EE. UU., África e, incluso, Rusia. Su aparición en el concierto bélico de Medio Oriente ha estado complementada por agresivas campañas comerciales para reclutar adeptos en más de cien países, quienes se convierten en guerreros suicidas. Esto se debe a su estrategia de "células dormidas" y "lobos solitarios", que han causado sangrientos atentados y ha dejado una costosa estela de mortandad, destrucción e incertidumbre en el mundo. El propósito geopolítico de ISIS es crear un califato como primera autoridad religiosa del mundo musulmán, para tener el control de los países, imponer la ley islámica, o "Sharia", y un estilo de vida musulmán radical.

En el otro extremo, se encuentran las milicias rebeldes, conformadas por más de 800 grupos; en su mayor parte, estos son afectos a los países occidentales y otros con apoyo logístico de $\mathrm{Al}$ Qaeda, al régimen de Ál-Asad y a milicias yihadistas radicales. El número de efectivos militares puede sobrepasar los 40.000 efectivos, muchos de ellos con suficiente experticia y conocimiento de las ubicaciones geográficas, las cuales patrullan permanentemente y no obedecen directamente a fuerzas extranjeras, pero sí reciben apoyo militar para realizar diferentes actividades militares.

También se encuentran los kurdos en el escenario de combate, dispersos en varios países como Turquía, Siria, Irak e Irán. Poseen lazos de amistad y cooperación continua con los países occidentales, y tienen intereses legítimos de tener algún día un territorio propio, de ahí el interés geopolítico de participar en la confrontación bélica. Hay 400.000 sirios kurdos ubicados en el norte de Siria, organizados en su mayor parte en diversas agrupaciones guerrilleras, y están enfrentados ISIS con la ayuda permanente de EE. UU. en insumos militares y apoyo aéreo.

Los kurdos son una población flotante en Medio Oriente y solicitan con urgencia desde 1920, de acuerdo con el Tratado de Sèvres, un Estado autónomo, que estaría localizado entre las fronteras de Turquía, Siria e Irak. De manera unilateral y aprovechando el conflicto actual y el liderazgo del PKK, buscan lograr la independencia de Kurdistán, a pesar de ser tildado por EE. UU., Rusia, Turquía y la Unión Europea de ser un grupo terrorista. La figura 5 permite visualizar en el recuadro la ubicación geográfica del Kurdistán. 
En conclusión, es claro que el mayor foco de tensión en el mundo es Medio Oriente, pues allí hay una diversidad de países con intereses heterogéneos, múltiples propósitos y expectativas, con atomización de poderes, fragmentación de territorios en donde muchos creen tener poder y ninguno tiene poder. ISIS alcanzó a dominar un amplio territorio de Siria e Irak, pero con la llegada de las grandes potencias a finales del 2015 y la posterior arremetida en lo corrido del 2016, este perdió más buena parte del territorio conquistado, incluidos bastiones de gran importancia en Siria como Ramadi, Raqqa y Palmira (principal bastión militar y centro de comunicaciones con demás enclaves geográficos cercanos), y Mosul, en Irak, eventos sucedidos luego de diferentes ataques de ISIS en Europa.

El complejo problema del Medio Oriente ha originado a su vez una crisis migratoria de grandes proporciones desde Siria hacia diferentes países, entre ellos varios europeos. Al mismo tiempo hay una incubación de "células dormidas" en Europa que mucho daño pueden causar y que incluso pueden generar enfrentamientos mayores entre las grandes potencias por el control del Medio Oriente. Por ahora y como medida de extrema urgencia, ISIS pierde territorios conquistados e influencia militar, pero gana capacidad de impactar y desestabilizar sociopolíticamente, especialmente a Europa, a través del terrorismo y la incertidumbre traducidos en varios atentados que han causado numerosas víctimas de varias nacionalidades.

Es este el nuevo escenario que jugará ISIS en adelante utilizando esta vez las famosas "células dormidas". El crecimiento inusitado de practicantes del islam en Europa, según reseña The Economist, hará que en un periodo de 50 años esta sea la religión monoteísta mayoritaria ("Islam in Europe", 2015). Este es un escenario sin lugar a dudas complejo para los estados europeos, asiáticos y de Norteamérica, los cuales deben contener la amenaza latente a su seguridad interviniendo militarmente en dos frentes: por un lado, los focos de tensión en el norte de África y Medio Oriente; por otro lado, en su propio territorio, pues, en países como Francia, Italia, Alemania y Reino Unido, el número de europeos adeptos al islam crece de forma exponencial, y aunque en Siria e Irak, la presencia militar se ha reducido notoriamente, la incertidumbre de posibles ataques continúa siendo latente en todo el mundo. En la figura 6 se puede analizar la magnitud del fenómeno descrito: 


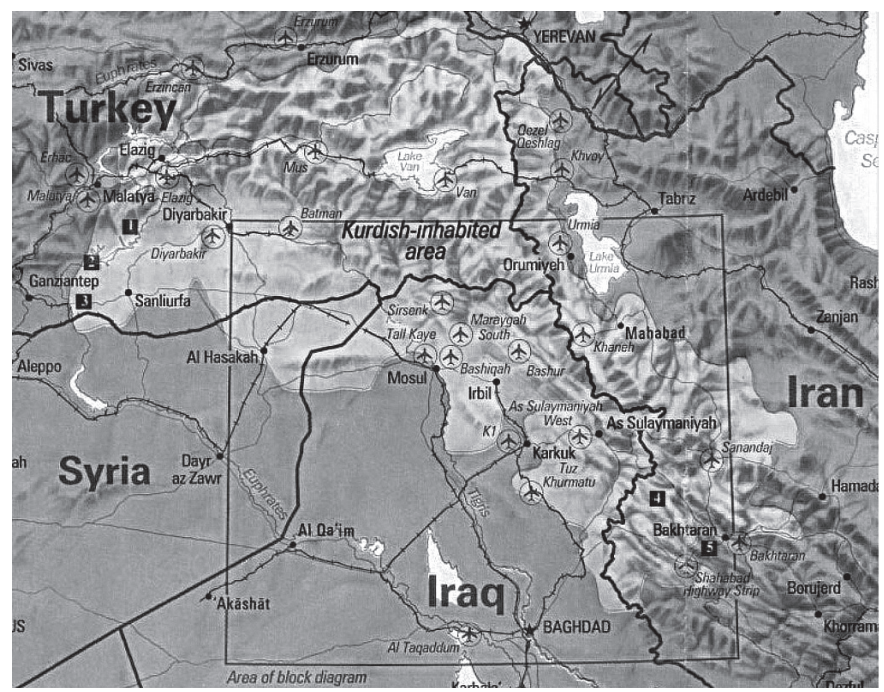

Figura 5. Kurdistán

Fuente: Open Democracy.

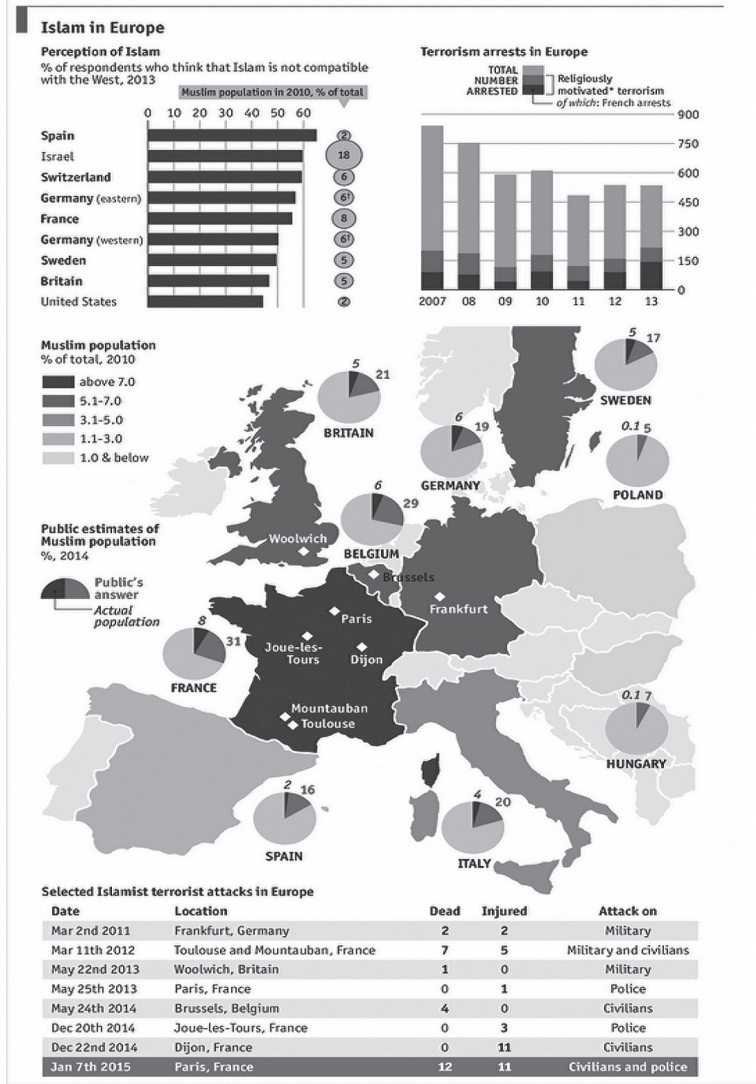

Figura 6. "Islam in Europe", 2015.

Fuente: The Economist. 


\section{Brexit: inicio de la fragmentación de la Unión Europea}

El 23 de junio del 2016, los británicos votaron a favor del Brexit ${ }^{3}$, decisión que exacerbó, aún más, la incertidumbre en los mercados mundiales. Aún después del primer mes del evento, los británicos no se repusieron de su determinación y muchos de ellos deseaban que se repitieran las votaciones. Sin embargo, el embajador británico ante la Unión Europea, sir Tim Barrow, entregó cinco días más tarde al presidente del Consejo Europeo, Donald Tusk, una carta escrita a mano por la primera ministra Theresa May, informándole que el Reino Unido había determinado separarse de la Unión Europea (UE), invocando el artículo 50 del Tratado de Lisboa, que dice que todo miembro de la UE "podrá decidir, de conformidad con sus normas constitucionales, retirarse" del bloque continental.

Es de destacar que el continente europeo, en su conjunto, es valorado como la primera potencia económica del globo con el 17,2\% del comercio mundial y 500 millones de personas, superando a la población norteamericana la cual es de 320 millones. El $45 \%$ de las exportaciones británicas van directamente a la UE. La decisión del Brexit obliga a que el Reino Unido en adelante deba negociar nuevos tratados de comercio con cada bloque económico y con cada uno de los países que demuestren perspectivas comerciales.

Al respecto, el Brexit tiene variados defensores como detractores. Para estos últimos, el Reino Unido nunca ha sido parte al cien por ciento del proyecto de la UE, pues sus habitantes y gran parte de gobernantes siempre han visto más desventajas que ventajas con la pertenencia al grupo; sin embargo, siempre ha existido una fuerte dependencia económica entre los dos actores, aspecto que forzó a la UE a reunir al presidente del Consejo Europeo, Donald Tusk, y al que era primer ministro británico, David Cameron, en el 19 de febrero del 2016 para analizar obligaciones propias de los países de UE. Para algunas de estas, Cameron solicitaba que fuera exceptuado el Reino Unido, como la restricción a trabajadores extranjeros en políticas sociales con menos de 4 años de residencia;

3 Brexit es una abreviatura entre Britain y Exit, con la cual se ha designado la escisión del Reino Unido de la Unión Europea en junio de 2016. 
también pedía que la ue reconociera la libra esterlina como moneda, además del euro, y que hubiera un tratamiento especial para el Reino Unido en relación con aquellas leyes que fueran en detrimento y debilitaran la posición competitiva de Reino Unido frente a India o China que crecen más que la zona euro. El análisis condujo a dar a los británicos un estatus especial en el seno de la UE.

En julio del 2016, las últimas proyecciones económicas del FMI redujeron las posibilidades de crecimiento de la economía mundial a la cifra de $3,1 \%$ para ese año, y a 3,4\% para el 2017. Se advertía un crecimiento para la economía latinoamericana de $-0,4 \%$, como consecuencia directa del Brexit, pues según el FMI: "El voto a favor del Brexit implica un aumento sustancial de la incertidumbre económica, política e institucional, la cual se proyecta que tenga consecuencias macroeconómicas negativas, sobre todo en las economías avanzadas de Europa" ("El FMI reduce su pronóstico de crecimiento mundial por el brexit y advierte de los riesgos para las perspectivas", 2016).

El Real Instituto Elcano ${ }^{4}$, entidad española que realiza diversos análisis y apreciaciones de geopolítica, utiliza desde el 2005 una herramienta empresarial denominada el Índice Elcano de Oportunidades y Riesgos estratégicos a partir de dos factores de alta relevancia: el índice de interdependencia económica y el índice de riesgo económico y político para todas las demás economías del mundo. Los dos elementos permiten configurar un mapa o radar de oportunidades y riesgos estratégicos, que refleja la importancia y nivel de riesgo de los 35 países del mundo con los que la economía británica mantiene relaciones económicas más intensas. Según el Índice Elcano, en el 2014, 23 países fueron los destinatarios del 82,3\% de las exportaciones de bienes del Reino Unido, al tiempo que sus importaciones procedieron también en un $88 \%$ de 23 países. Para una economía como la británica, cuyo valor del PIB anual es cercano a los tres billones de dólares, el daño económico por la ruptura podría ser letal, especialmente para el sector financiero,

4 Para remitirse a la página del Instituto el lector puede consultar el siguiente enlace: http://www.realinstitutoelcano.org/wps/portal/web/rielcano_es/!ut/p/c5/04_SB8K8xL LM9MSSzPy8xBz9CP0os3jjEBf3QG93QwMDyyBnA0d3C-8QS1NDgzAPE6B8pFm8AQ7g aEBAt59Hfm6qfkFuRDkAUzSsyA!!/d13/d3/L2dBISEvZ0FBIS9nQSEh/ 
el cual contaba con planes de contingencia. En el radar sobresalen oportunidades de negocios con países como Luxemburgo, Países bajos, Noruega, Alemania, Francia, Suiza, Bélgica, Polonia y República Checa, todos ellos pertenecientes a la UE.

Luego de un año del Brexit, los resultados no son alentadores. Las elecciones parlamentarias que se llevaron a cabo el 8 de junio del 2017 debilitaron la posición política del partido conservador, que, aunque todavía conserva mayorías en el parlamento inglés, es suficiente para lograr mayoría. No obstante, logró un acuerdo con el partido democrático de Irlanda que le permitía tener una mayoría minoritaria.

\section{Conclusiones}

Caos, volatilidad y tensión son los rasgos cada vez más predominantes en el orden político y económico global. Esta es la oportunidad para destacar a la geopolítica como una disciplina que permite la construcción de perfiles de análisis más completos e integradores a partir de las diferentes metodologías y áreas del conocimiento que la complementan y que hacen de ella un saber que está en constante actualización: es la geografía en movimiento. Desde este punto de vista es necesario subrayar la importancia creciente que adquiere como herramienta de interpretación, análisis y explicación de los fenómenos y la conflictividad del mundo de hoy y la necesidad de integrarla a los circuitos académicos colombianos.

En ese orden de ideas, el presente artículo se constituye como una herramienta de consulta para los empresarios, académicos e interesados en la búsqueda de elementos que permitan la comprensión del orden geopolítico actual y la toma de decisiones alrededor de situaciones políticas y comerciales que ameriten una lectura integral del contexto en el que se desenvuelve cada actor y que sean de utilidad para determinar necesidades, objetivos y resultados.

Es claro que el análisis y la discusión no se agotan en este ejercicio, los cambios ejercidos por la administración de Donald Trump en materia ambiental todavía están por surtir efecto, lo que hace más complejo tratar de establecer proyecciones al respecto. Así mismo, la radicalización de su discurso frente a Corea del 
Norte y la repuesta por parte del régimen de Pyongyang generan alarmas de guerra el mundo. Adicionalmente, las implicaciones geopolíticas de procesos como el Brexit, el mar meridional de China y la crisis de refugiados en Medio Oriente son prematuras de establecer por lo reciente de dichos acontecimientos. Por último, este artículo como insumo académico invita a los lectores más interesados por el análisis geopolítico a profundizar o complementar en las temáticas abordadas en el documento y proponer nuevas líneas de investigación que fortalezcan la producción intelectual y bibliográfica en la materia.

La invitación está abierta.

\section{Referencias}

Ballesteros Marín, M. A. (2013). La evolución de los conflictos. Panorama Geopolítico de los Conflictos. Madrid: Instituto de Estudios Estratégicos del Gobierno Español.

Ballesteros Marín, M. A. et al. (2014). Panorama geopolítico de los conflictos 2016. Madrid: Instituto de Estudios Estratégicos del Gobierno Español.

Castells, M. (1998). Globalización, tecnología, trabajo, empleo y empresa. Barcelona: Alianza Editorial.

Chomsky, N. (2007). El nuevo orden mundial (y el viejo) (Carme Castells, tr., 5. ${ }^{\text {a }}$ edición), Barcelona: Crítica.

Claval, P. (1999). El enfoque cultural y las concepciones geográficas del espacio. Boletín de la AGE, (34), 21-39

Dallanegra Pedraza, L. (2008). El árbitro supremo: el problema del 'gobierno' del mundo. Revista Círculo de Humanidades, 52, 1-28

El FMI reduce su pronóstico de crecimiento mundial por el brexit y advierte de los riesgos para las perspectivas. (2016). FMI. Recuperado de http:// www.imf.org/es/News/Articles/2016/07/18/18/11/NA07192016-IMFCuts-Global-Growth-Forecasts-on-Brexit-Warns-of-Risks-to-Outlook

Islam in Europe. (2015). The Economist. Recuperado de https://www. economist.com/blogs/graphicdetail/2015/01/daily-chart-2

Hernández Alvarado, J. (2002) Geopolítica del conflicto: el mundo después del 11 de septiembre. Revista Iconos de Ciencias Sociales Flacso,13, 124-129.

Hobsbawm, E. (1997). Las hegemonías de Estados Unidos y Gran Bretaña. Revista Estudios Políticos, 39, 1-11.

Hobsbawm, E. (1998). La era del Imperio 1875-1914. Barcelona: Crítica.

Hobsbawm, E. (1999). Primer y tercer mundo después de la guerra fría. Revista Cepal, 67, 7-14. 
Lacoste, Y. (2008). Geopolítica: la larga historia del presente. Barcelona: Síntesis. Luttwak, E. (1990) From geopolitics to geoeconomics: Logic of conflict, grammar of commerce. The National Interest, 20, 17-23.

Méndez, R. (2011). Tensiones y conflictos armados en el sistema mundial: una perspectiva geopolítica. Revista de Investigaciones Geográficas, 55, 19-37.

Olier, E. (2011). Geoeconomía: las claves de la economía global. Madrid: Prentice Hall.

Raffestin, C. (2011). Por una geografía del poder. Michoacán. Editorial Colegio de Michoacán.

Ratzel, F. Las leyes del crecimiento espacial de los Estados. Geopolíticas, 2, $135-156$

Sanguin, A. (1981) Geografía política. Barcelona: Oikos Tau.

Wallerstein, I. (2005). Análisis de sistemas-mundo: una introducción. Ciudad de México: Siglo XXI. 